Journal of the Korean Physical Society, Vol. 73, No. 5. no, Month 2018, pp. 531-537

\title{
Relativistic Treatment of Spin-zero Particles Subjected to the Shifted Tietz-Wei Potential Model
}

\author{
C. A. Onate
}

Physics Programme, Department of Physical Sciences, Landmark University, Omu-Aran, Nigeria and

Department of Physics, University of Benin, Nigeria

A. N. Ikot * and M. C. Onyeaju

Theoretical Physics group, Department of Physics, University of Port Harcourt, P.M.B. 5323 Choba Port Harcourt, Nigeria

E. E. Ituen

Theoretical Physics group, Department of Physics, University of Uyo, Nigeria

E. Aghemenloh and O. Ebomwonyi

Department of Physics, University of Benin, Nigeria

(Received 30 August 2017, in final form 9 February 2018)

Abstract

The approximate solutions of the Klein-Gordon equation with the shifted Tietz-Wei potential function are thoroughly studied for Klein-Gordon equation with both $2 V$ and $V$ potentials by usinga suitable approximation scheme for the centrifugal term in the framework of the supersymmetric approach. The non-relativistic limits in both cases are obtained, and the numerical results are computed. We have equally investigated the Fisher information on the shifted Tietz-Wei potential function in the case of the non-relativistic limit of the Klein-Gordon equation with potential $V$ because this is only possible in the non-relativistic regime. The Fisher information is observed to be inversely proportional to both $C h$ and $b h$, where $C h$ is the optimization parameter and $b h=\beta(1-C h)$, with $\beta$ being the Morse constant.

PACS numbers: 03.65.Fd, 03.65.Pm, 03.65.Ca, 03.65.Ge

Keywords: Klein-Gordon equation, Eigensolutions, Wave equations, Fisher information DOI: $10.3938 / \mathrm{jkps} .72 .0$ 